\title{
Recipient outcomes of kidney transplantation from older living donor
}

Ji-Yeon Song, Kyo-Won Lee

Department of Surgery, Samsung Medical Center, Seoul, Korea

Background: As a solution to the shortage of organ supply, studies on transplantation from older kidney donor are being conducted. Still, there are many controversies about safety and efficacy about this, but sufficient research has not been made. Methods: In Samsung Medical Center, from 2000 January to 2015 May, total 906 kidney transplantation cases were elected by inclusion and exclusion criteria and analyzed by dividing into two groups according to the age of the donor: younger group (donor age, $18-59$ years) and older group (donor age, $\geq 60$ years).

Results: There was no significant difference between the two groups in patient death and postoperative complications. Older donor group had poorer acute rejection rate $(P=0.028$; hazard ratio $[H R], 1.733)$, graft failure rate $(P=0.032 ; H R, 2.31)$, and 10 -year estimated glomerular filtration rate (eGFR; 77.75 vs. $60.30, P=0.015$ ). There was no significant difference between the two groups in the trend of eGFR change over time.

Conclusions: In terms of safety, there is no disadvantage in renal transplantation through an elderly donor. In terms of efficacy, poorer acute rejection, graft failure rate, and 10-year eGFR suggest that kidney transplantation through elderly donors should be carried out carefully, whereas the fact that there is no difference in the trend of eGFR change over time can lead to a conclusion that it is not inferior in efficacy either. Therefore, larger studies will need to be conducted to accurately analyze the efficacy of the elderly kidney transplant.

Corresponding author: Kyo-Won Lee

E-mail: kw1980.lee@samsung.com

\section{(C) The Korean Society for Transplantation}

This is an Open Access article distributed under the terms of the Creative Commons Attribution Non-Commercial License (http://creativecommons.org/licenses/by-nc/4.0/) which permits unrestricted non-commercial use, distribution, and reproduction in any medium, provided the original work is properly cited. 